\title{
Imaginando futuros distintos. Los efectos de la desigualdad sobre las transiciones hacia la educación secundaria posobligatoria en la ciudad de Barcelona
}

\author{
Judith Jacovkis \\ Alejandro Montes \\ Martí Manzano \\ Universitat Autònoma de Barcelona \\ judith.jacovkis@uab.cat; alejandro.montes@uab.cat; marti.manzano@uab.cat
}

Recepción: 17-10-2019

Aceptación: 20-12-2019

Publicación: 01-04-2020

\section{Resumen}

Las transiciones a la educación secundaria posobligatoria son un momento clave en la trayectoria educativa de los jóvenes. Las decisiones que los llevan a seguir unos u otros estudios son producto de una agencia delimitada por condicionantes estructurales (Furlong, 2009). Así, las desiguales posiciones sociales son fundamentales para explicar diferencias en su experiencia escolar, sus horizontes de futuro, sus expectativas y sus aspiraciones (Gillborn et al., 2012).

Partiendo de este marco, este artículo analiza el impacto de variables de la estructura social y del sistema educativo en la configuración de las expectativas y aspiraciones educativas de jóvenes que acaban de transitar hacia la educación secundaria posobligatoria en la ciudad de Barcelona. El análisis explota las respuestas de un cuestionario distribuido durante el curso 2018-19 a una muestra de alumnado $(\mathrm{N}=1.318)$ del primer curso de Bachillerato y de Ciclos Formativos de Grado Medio en ocho institutos de la ciudad de Barcelona.

Los principales resultados constatan la desigualdad en los perfiles del alumnado que accede a una u otra modalidad de estudios secundarios posobligatorios. Además, profundizan en la comprensión de la interacción y del impacto de elementos estructurales y del sistema educativo sobre la definición de escenarios de futuro desiguales entre el alumnado que sigue la vía académica o la vía profesional, y entre el alumnado de distinto perfil social. Palabras clave: educación secundaria posobligatoria; transiciones educativas; formación profesional; bachillerato; desigualdades educativas; expectativas; aspiraciones 
Abstract. Imagining different futures: The effects of inequality on transitions to post-compulsory secondary education in the city of Barcelona

Transitions to post-compulsory secondary education are a crucial moment in the educational trajectory of young people. The decisions that lead them to pursue different educational alternatives are the product of an agency delimited by structural constraints (Furlong, 2009). Thus, their unequal social positions are fundamental to explain differences in their school experience, their future horizons, their expectations and their aspirations (Gillborn et al., 2012). Based on this framework, the paper analyses the impact of variables in the social structure and education system on the configuration of educational aspirations and expectations of young people who have just moved on to upper secondary education in the city of Barcelona. The analysis exploits responses to a questionnaire distributed during the 20182019 school year in eight high schools of Barcelona to a sample of students $(\mathrm{N}=1,318)$ in their first year of post-compulsory education, in both the vocational and academic track. The main results of the analysis confirm the inequality in profiles of students who access each post-compulsory track. In addition, the findings deepen our understanding of the interaction and impact of structural elements and the educational system on the definition of unequal future scenarios among students following the academic and vocational tracks, and among students from different social backgrounds.

Keywords: post-compulsory secondary education; educational transitions; vocational education and training; baccalaureate; educational inequalities; expectations; aspirations

\author{
Sumario \\ 1. Introducción 5. Resultados \\ 2. Marco teórico 6. Conclusión \\ 3. Contexto Financiamiento \\ 4. Metodología Referencias bibliográficas
}

\title{
1. Introducción
}

El proceso de transición a la educación secundaria posobligatoria supone un momento clave en la configuración de las trayectorias educativas y laborales de los y las jóvenes. Los momentos de transición, en el sistema educativo y hacia el mercado de trabajo, pueden entenderse como procesos en los que factores institucionales, socioeconómicos e individuales interactúan para contribuir en la definición de trayectorias educativas (Cuconato y Walther, 2015). Las transiciones, en este sentido, no son neutrales en términos de clase, género ni etnia, y tampoco son independientes de los contextos institucionales en los que se desarrollan (Walther, 2006). En contextos institucionales como el de Barcelona - y el de España en general_- en este momento de transición no solo se abandona la etapa de enseñanza obligatoria, sino que, además, se definen dos itinerarios formativos, el académico y el profesional, entre los cuales el alumnado elige. Estas elecciones, como el propio proceso de transición, no se realizan en el vacío, sino que son producto de una agencia delimitada por 
condicionantes estructurales (Tikkanen et al., 2015; Furlong, 2009). Así, las posiciones sociales — desiguales - de los estudiantes son fundamentales para explicar diferencias en su experiencia escolar, en sus imaginarios de futuro, en sus expectativas y en sus aspiraciones (Gillborn et al., 2012). De hecho, como evidencian diversas investigaciones en el ámbito internacional (Blossfeld y Shavit, 2011) y en el estatal (Bernardi y Requena, 2010; Calero, 2008), las transiciones entre etapas educativas, y especialmente de la educación obligatoria a la posobligatoria, son momentos clave en términos de selectividad social.

Partiendo de este marco, el artículo tiene como objetivo analizar el impacto de variables de la estructura social y del sistema educativo en la configuración de las aspiraciones y expectativas educativas de jóvenes que acaban de transitar hacia la educación secundaria posobligatoria en la ciudad de Barcelona. En este sentido, el texto se interroga sobre la interacción y la influencia de las variables de itinerario formativo, clase social, género y origen migratorio en la configuración de futuros aspirados y esperados por parte del alumnado de primer curso de la educación secundaria posobligatoria. Para ello, el texto se divide en cinco apartados. El primero desarrolla el marco teórico que orienta el análisis. En él se explora la literatura sobre transiciones y trayectorias educativas, y sobre expectativas y aspiraciones de los jóvenes. A través de esta revisión de la literatura, se pone de manifiesto el carácter social y construido de las expectativas y las aspiraciones, y se discuten planeamientos individualizadores o psicologicistas de las mismas. El segundo destaca algunas de las características principales del contexto institucional en el que se enmarca el tránsito de la educación secundaria obligatoria a la posobligatoria, así como algunos datos que contribuyen a describir el campo de análisis de la ciudad de Barcelona. El tercero presenta la metodología de análisis y las técnicas de recolección de datos utilizadas. El cuarto expone los resultados del análisis. Por un lado, detalla las características de la muestra en términos de su distribución en los itinerarios académico y profesional. Por el otro, explora las diferencias y las similitudes en los horizontes de futuro del alumnado en función del itinerario educativo elegido y de su perfil social. Finalmente, el quinto apartado discute los resultados obtenidos y presenta las conclusiones del análisis.

\section{Marco teórico}

\subsection{Cambios y continuidades en las trayectorias vitales y educativas}

Las pautas que caracterizan las trayectorias vitales y educativas de los y las jóvenes han cambiado en las últimas décadas. Como señalan Wyn y Dwyer (2002), la linealidad propia de las trayectorias juveniles en el período posterior a la Segunda Guerra Mundial se ha alterado en diversas direcciones. La relación entre escuela y trabajo ha perdido secuencialidad, y las entradas y salidas del mercado laboral y de las instituciones educativas son mucho más frecuentes de lo que solían ser. Las transiciones, como han captado Biggart y Walther (2005) con la expresión de «trayectorias yo-yo», pueden entenderse 
mejor como oscilaciones entre la juventud y la adultez, entre la autonomía y la dependencia, entre la educación y el trabajo. En este contexto, los mismos autores han considerado que las trayectorias vitales de los y las jóvenes se han desestandarizado, $y$ han desdibujado de algún modo lo que hasta el momento se consideraba como una trayectoria «normal».

Esta desestandarización, sin embargo, no ha supuesto necesariamente un aumento de las oportunidades para ellos. Como señalan Cuconato y Walther (2015), el crecimiento de discursos fuertemente «agenciales», que magnifican la potencialidad del individuo para elegir y construir su propia trayectoria vital, contrasta con la precariedad del mercado de trabajo al que se accede después de los estudios. Ello incrementa la discordancia entre las expectativas por el aumento del nivel de estudios y la realidad de los puestos de trabajo disponibles (Wyn y Dwyer, 2002). Este contraste, de hecho, muestra la tensión entre el debilitamiento de "lo colectivo" y el énfasis discursivo en las salidas individuales a las situaciones de precariedad (Brunila et al., 2011).

Los cambios en los contextos que marcan las trayectorias vitales de los y las jóvenes han llevado a diversos autores a privilegiar los análisis centrados en la agencia y la reflexividad de los sujetos frente a aquellos de carácter más estructural. Estos análisis han considerado que los factores vinculados a la posición socioeconómica de los jóvenes han perdido relevancia a la hora de explicar y entender cómo se definen los cursos de vida (Beck, 1992). Por otro lado, la tendencia política a nivel europeo enfatiza la importancia de seguir estrategias de activación que permitan a los jóvenes expandir sus oportunidades (Rinne et al., 2019). Con ello, como señalan diversas investigaciones, se pone el acento en la responsabilidad individual más que en la capacidad de las instituciones de dar respuesta a las necesidades de los jóvenes (Rodríguez-Soler y Verd, 2018; Serrano y Martín, 2017; Walther, 2006).

Sin embargo, como ha subrayado Furlong (2009), y como muestra la evidencia que sintetizamos a continuación, las transformaciones experimentadas en las últimas décadas no restan relevancia a los condicionantes institucionales y socioeconómicos como factores explicativos de diferencias y desigualdades en las trayectorias educativas. Estas desigualdades, además, darán lugar posteriormente a diferencias y desigualdades en las oportunidades de futuro de los y las jóvenes. De hecho, la investigación comparada ha permitido analizar de qué manera los entramados institucionales en general y la arquitectura de los sistemas educativos en particular impactan sobre la definición de trayectorias educativas. Tikkanen et al. (2015) analizan cómo distintas arquitecturas institucionales configuran estructuras de oportunidad y elección desiguales. Así pues, la facilidad o la dificultad para acceder a una vía formativa u otra, las oportunidades que cada titulación brinda en términos de continuidad educativa o los costes asociados a la educación, entre otros, son factores centrales para explicar la configuración de trayectorias educativas desiguales (Tarabini y Jacovkis, 2019b). Como señala Walther (2006), distintos regímenes de transición impactan de forma diferenciada sobre la formación de subjetividades y de biografías individuales, de las que las elecciones forman parte. 
Si nos centramos específicamente en la transición de la educación secundaria obligatoria a la posobligatoria, la investigación realizada en el contexto español muestra claras desigualdades de tipo socioeconómico en la participación, en la distribución entre itinerarios y en los resultados que se obtienen en esta etapa. Con relación al acceso, Calero (2008) señala las diferencias abismales entre el porcentaje de alumnos de clase media y el de los de clase trabajadora que acceden a algún tipo de educación secundaria posobligatoria $(85,3 \%$ los primeros y 52,2\% los segundos). ${ }^{1}$ Por otro lado, atendiendo a la distribución del alumnado según el itinerario formativo, a igualdad de rendimiento en la etapa obligatoria, los estudiantes de clase trabajadora tienen más probabilidades de seguir la vía profesional que los de clase media (Bernardi y Requena, 2010). Además, como indican Elias y Daza (2017), la desigualdad educativa también afecta a las calificaciones, que tienen una influencia clara en la configuración de las expectativas, de las aspiraciones y del autoconcepto del alumnado. Por lo tanto, no solo el entramado institucional impacta en la configuración de trayectorias educativas específicas, sino que lo hace de forma diferencial según la posición que los y las jóvenes ocupan en la estructura social.

Para dar cuenta de cómo la posición en la estructura social contribuye en la configuración de las elecciones y las trayectorias educativas, la literatura incorpora factores culturales y sociales, como son el prestigio de los itinerarios educativos o su asociación con un tipo u otro de alumno. Por un lado, la evidencia demuestra que existen atribuciones de prestigio e idoneidad que tienden a vincular mejores resultados académicos en la etapa obligatoria con una mayor aptitud para seguir con el itinerario académico, y peores resultados académicos con facilidades para realizar tareas manuales. En este sentido, las habilidades manuales se vinculan al campo de conocimiento propio de las clases trabajadoras, mientras que las habilidades intelectuales se relacionan con las clases medias (Lahelma, 2009). En clave de género, Lappalainen et al. (2013) incorporan las prácticas de orientación —y sus supuestos— al análisis de las elecciones educativas. En la misma línea, Brunila et al. (2011) analizan las desigualdades de género en las transiciones a la educación posobligatoria centrándose en las barreras culturales hacia la formación profesional por parte de determinados colectivos, y en la construcción de la vía académica como itinerario obvio o normalizado. Por su parte, Gillborn et al. (2012) constatan que los alumnos pertenecientes a minorías étnicas también se ven afectados por sesgos en la orientación y en las expectativas docentes que contribuyen a reproducir la posición que ocupan en la estructura social. En este sentido, se identifica el origen migratorio como un factor mediador clave en la construcción de expectativas y aspiraciones de jóvenes que han migrado o que descienden directamente de migrantes (Kilpi-Jakonen, 2011).

1. Cabe destacar que el primer porcentaje - 85,3\% — corresponde a profesionales, y el segundo - 52,2 \%- a trabajadores manuales cualificados. Si nos fijamos en los trabajadores no cualificados, el porcentaje cae hasta el $27,5 \%$, de manera que lo distancia en casi 30 puntos de la tasa media, situada en el 56,9\% (Calero, 2008: 53). 
Así pues, la literatura confirma la relevancia de los factores socioeconómicos y culturales en el análisis de las trayectorias educativas, y pone en entredicho la fundamentación de discursos centrados en las capacidades individuales como elementos definitorios del éxito educativo. En las próximas líneas, se presentan algunas aportaciones que, a través del análisis de la construcción — desigual— de las expectativas y las aspiraciones del alumnado, pueden contribuir a mejorar nuestro conocimiento sobre los mecanismos que conectan las trayectorias educativas de los y las jóvenes con su perfil socioeconómico.

\subsection{La desigual construcción de expectativas y aspiraciones socioeducativas}

Son muchos los autores que han trabajado en el terreno de las aspiraciones y las expectativas (Gale y Parker, 2015; Boxer, et al., 2011; Dubow et al., 2009), con la idea de establecer las bases para su conceptualización y delimitación. Según las aportaciones de estos trabajos, las aspiraciones refieren al imaginario deseado, mientras que las expectativas tienen una base más terrenal y hacen referencia a las previsiones o posibilidades asumidas para un futuro próximo. En esta línea, Markus y Nurius (1986) introdujeron el concepto de "possible selfss a la hora de identificar desconexiones o diferencias entre las aspiraciones y las expectativas de un mismo individuo. Para estos autores, las aspiraciones son representaciones cognitivas construidas de acuerdo con autoproyecciones futuras, no necesariamente ancladas en la realidad social. En cambio, las expectativas se basan en proyecciones ancladas en los elementos estructurales de la realidad social, y conectan con los factores facilitadores y dificultadores percibidos en el entorno social más próximo (Markus y Nurius, 1986; Armstrong y Crombie, 2000). Por su parte, Hegna (2014) y Hodkinson y Sparkes (1997) profundizan en el análisis del rol que juegan los elementos estructurales en la definición de las autoproyecciones. Así, definen las aspiraciones educativas como el reflejo de las percepciones subjetivas en torno a las capacidades personales y a las oportunidades externas. Estas, a su vez, están influenciadas por las características sociales de los y las jóvenes (el género, la etnia o el perfil socioeconómico). En esta línea, estos autores concluyen que las expectativas y las aspiraciones no pueden comprenderse si no es en relación con los contextos institucionales y «vitales» de los jóvenes (Hodkinson y Sparkes, 1997; Hegna, 2014).

Los distintos grupos sociales imaginan sus proyecciones futuras, deseables y posibles, de manera claramente diferente (Khattab, 2015). La plasmación de las expectativas educativas o, dicho de otro modo, el nivel educativo que los jóvenes esperan alcanzar son altamente divergentes para los diferentes grupos sociales (Hegna, 2014). Esta divergencia proviene de los elementos estructurantes propios de cada joven (posición socioeconómica, sociocultural o biográfico-personal, entre otros elementos). En este sentido, tal y como destaca Curran (2017: 84), podríamos afirmar que las expectativas «son un reflejo de las desigualdades sociales existentes».

Centrándonos en los factores que afectan a esta construcción desigual de imaginarios de futuro, en primer lugar, se observa una clara influencia de las 
desigualdades sociales en la brecha existente entre los itinerarios formativos asumidos como "propios», esperados, y las opciones educativas y profesionales deseadas o aspiradas. Para autores como Furlong y Biggart (1999), dicha brecha va creciendo y haciéndose más explícita conforme avanza la trayectoria educativa del o la joven, y se impone sutilmente como si de una realidad objetiva se tratase. Bourdieu (1973) definiría este fenómeno como la internalización de las probabilidades objetivas de éxito. Para Archer et al. (2007), esto provoca que los jóvenes provenientes de entornos socioeconómicos desfavorecidos se sometan a un procedimiento de autoexclusión basado en la percepción de que determinado tipo de estudios posobligatorios no encaja o no está hecho para "gente como ellos». Además, la autora señala cómo estas restricciones operan intangiblemente a través de la violencia simbólica, que, en términos de Bourdieu, es la que genera la reproducción de las propias condiciones de subordinación.

En segundo lugar, se observa que los jóvenes de entornos sociales más desfavorecidos son sometidos a un claro procedimiento de reducción de las aspiraciones por parte de la institución escolar (Dupriez, et al., 2012; Romito, 2017). Por su parte, estos jóvenes abrazan esta lógica pues perciben que sus oportunidades disponibles son decrecientes y adaptan sus aspiraciones a tal percepción (Ball, et al., 2002). Además, este fenómeno refuerza la idea expuesta por Romito (2017) sobre el papel del orden escolar y de los mecanismos de orientación y acompañamiento educativo en la regulación, en sus propios términos, del desarrollo de determinadas trayectorias. En este sentido, el papel jugado por la institución escolar refuerza la interiorización, principalmente por parte del alumnado que no concuerda con los estándares académicos, de un conjunto de subjetividades que operan en la línea de legitimar el desarrollo de trayectorias «supuestamente más adecuadas», lo que refrenda el proceso de reducción de las aspiraciones educativas. Del mismo modo, Hegna (2014) refuerza estos elementos observando cómo, después de la transición a la educación secundaria, este proceso de disminución de las aspiraciones se agrava en aquellos casos en los que se producen bajas calificaciones y un rendimiento escolar por debajo de la media.

Así pues, la posición social de origen y el entorno sociofamiliar juegan un papel fundamental a la hora de definir, consciente e inconscientemente, las aspiraciones de los y las jóvenes, puesto que los individuos construyen su trayectoria educativa (y articulan las diferentes transiciones) basada en horizontes particulares, establecidos de acuerdo con sus antecedentes de clase social, género y origen étnico (Hodkinson y Sparkes, 1997). De hecho, como muestra la investigación en este campo (Gale y Parker, 2015; Archer, et al., 2007; Hegna, 2014), es necesario destacar que la capacidad de construcción de «futuros deseados» se distribuye de forma desigual entre los y las jóvenes en función del grupo social al que pertenecen.

En definitiva, y en términos de Boxer et al. (2011: 610), «las aspiraciones y expectativas son, por lo tanto, influencias potencialmente críticas en las futuras trayectorias académicas y ocupacionales de los jóvenes. Si bien, por supuesto, 
ni las aspiraciones ni las expectativas operan en el vacío». Por ejemplo, los estudios de Dubow et al. (2009) o de Beal y Crockett (2010) ponen de relieve la influencia de las expectativas, propias y familiares, sobre la importancia otorgada a la continuidad educativa, la percepción de adquisición de competencias, habilidades y valores escolares (y en consecuencia, en la conformación del autoconcepto) o la participación en actividades extracurriculares relacionadas con el ámbito académico. Asimismo, autores como Thrupp et al. (2002) enfatizan el «efecto de los pares» para explicar parte del contagio identificado en entornos homogéneos en los que predominan las bajas expectativas y el rechazo al sistema escolar. Dada esta vinculación entre expectativas, aspiraciones y mayor o menor adhesión a la institución escolar, no es de extrañar, entonces, que la mayoría de instituciones educativas internacionales, como la OCDE, haya apostado en los últimos años por mejorar las aspiraciones educativas de los y las jóvenes como estrategia para elevar las participación en la educación posobligatoria (Curran, 2017), así como para incrementar la competitividad económica (Gale y Parker, 2015).

\section{Contexto}

El sistema educativo español es formalmente comprensivo hasta la finalización de la Educación Secundaria Obligatoria (ESO). ${ }^{2}$ La transición a la educación secundaria posobligatoria, tal y como se comenta en la introducción, supone el primer momento de elección en la trayectoria educativa de los y las jóvenes. Así, una vez obtenido el graduado en ESO, el alumnado se enfrenta a la decisión de elegir un itinerario de carácter académico - Bachillerato- o uno de carácter profesional -Ciclo Formativo de Grado Medio (CFGM) (ver figura 1).

En la ciudad de Barcelona, el alumnado de las dos vías formativas se distribuye de forma desigual, como sucede en el resto de Cataluña. Así, para el curso 2018-19, el alumnado matriculado en $1^{\circ}$ de Bachillerato representa casi el $60 \%$ del total de la matrícula en el primer curso de la educación secundaria posobligatoria de la ciudad, mientras que el matriculado en CFGM supera por poco el $40 \%{ }^{3}$ Además, las características del alumnado en cada uno de los itinerarios son dispares en términos de género y origen migratorio. El alumnado extranjero representa un $11 \%$ del total de la matrícula en la educación secundaria posobligatoria. No obstante, su peso en los CFGM se acerca al $15 \%$, mientras que en Bachillerato no alcanza el $9 \%$. Por otro lado, las chicas representan alrededor del $42 \%$ del alumnado de primer curso de CFGM, mientras que el porcentaje de chicos supera el $57 \%$. En cambio, en

2. Es preciso señalar que, a pesar de que la norma define las etapas de escolarización obligatoria como comprensivas, existen prácticas de agrupación por niveles dentro de los centros. Como señalan Castejón y Pàmies, «según PISA 2015, un 26 \% del alumnado está escolarizado en centros de secundaria que agrupan a sus estudiantes por niveles en todas las asignaturas» (2018: 49).

3. Los datos de esta sección provienen de la «Estadística del Departament d'Ensenyament de Catalunya» para el curso 2018-19. 
Figura 1. Estructura del sistema educativo español

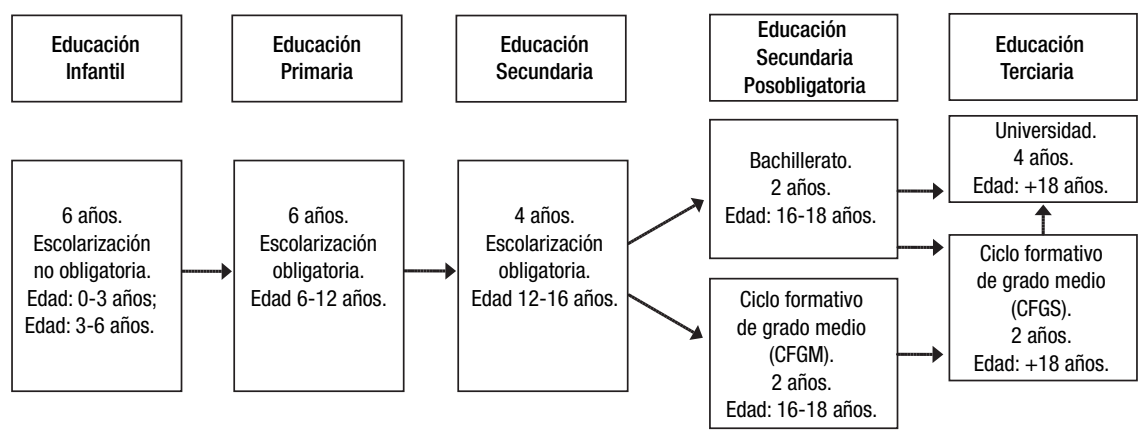

Fuente: adaptación de Tarabini et al. (2018).

Bachillerato, el alumnado femenino se sitúa cerca del $55 \%$, mientras que el masculino ronda el $45 \%$. Aunque en este trabajo no profundizamos sobre esta cuestión, es preciso señalar que la distribución del alumnado dentro de cada uno de los itinerarios formativos es claramente desigual en términos de género. Así, familias como Imagen Personal, Servicios Socioculturales y a la Comunidad y Sanidad están altamente feminizadas, mientras que otras como Electricidad y Electrónica, Emergencias y Protección Civil, Energía y Agua, Industrias Extractivas, Fabricación Mecánica, Informática y Comunicaciones, Instalación y Mantenimiento, Marítimo-pesquera y Transporte y Mantenimiento de Vehículos apenas cuentan con matrícula femenina (Departament d'Ensenyament, 2019).

Este breve repaso sobre las características del alumnado en cada una de las vías formativas pone de relieve la importancia de profundizar en el conocimiento sobre los factores que contribuyen a definir las elecciones que marcan la transición a la educación secundaria posobligatoria.

\section{Metodología}

Como se ha señalado, este artículo tiene por objetivo analizar las transiciones a la educación secundaria posobligatoria en la ciudad de Barcelona. En este caso, el foco se sitúa en explorar los sesgos sociales en las expectativas y aspiraciones de los estudiantes, que resultan en trayectorias educativas desiguales. Para realizar este análisis, el artículo se basa en los datos recogidos a partir de un cuestionario online que se distribuyó en ocho centros educativos de Barcelona con oferta tanto de Bachillerato como de CFGM. Los centros fueron seleccionados por ser diversos en su composición social, en su oferta y en su ubicación geográfica. Respecto a la composición social, seleccionamos centros con mayoría de alumnos de clases sociales acomodadas y medias, centros con clases trabajadoras y centros con alta concentración de alumnado en riesgo de exclusión. En cuanto a la oferta posobligatoria, elegimos centros con bachilleratos genéricos y espe- 
cíficos y un abanico de oferta de familias de CFGM. Finalmente, respecto a la ubicación geográfica, seleccionamos centros en zonas de la ciudad sobre la media del nivel de renta, centros en zonas que superan este nivel y centros en zonas con niveles inferiores a la media. Esta selección se diseñó con el objetivo de incluir las principales casuísticas en la oferta educativa posobligatoria de Barcelona y de garantizar cierto nivel de generalización de los resultados. ${ }^{4}$

El cuestionario se distribuyó entre todos los alumnos del primer curso de educación posobligatoria de los ocho centros, entre octubre y diciembre de 2018, es decir, en los primeros meses de sus estudios posobligatorios. Siempre se realizó en aulas del centro y bajo la supervisión de algún investigador o investigadora. Se obtuvieron un total de 1.318 respuestas, 697 (52,9\%) correspondientes a alumnos y alumnas de primero de Bachillerato, y $621(47,1 \%)$ a alumnos y alumnas de primero de CFGM.

Dentro del itinerario de Bachillerato, la muestra se distribuye por especialidades de la siguiente manera. En primer lugar, Humanidades y Ciencias Sociales (241 alumnos), seguida por Ciencia y Tecnología (220), Artístico (210) y, finalmente, Bachillerato Internacional y Bachibac (26). En los CFGM, la muestra por especialidades es, de mayor a menor número de alumnos: Sanidad (237), Imagen Personal (87), Transporte y Mantenimiento de Vehículos (62), Comercio y Marketing (56), Administración y Gestión (56), Informática y Comunicaciones (42), Actividades Físicas y Deportivas (41) y Servicios Socioculturales y a la Comunidad (40).

El cuestionario se divide en cuatro bloques. El primer bloque es de carácter introductorio y recoge datos como la edad, el género o la modalidad de estudios del alumnado. El segundo explora la trayectoria educativa previa de los y las jóvenes a nivel de resultados y de experiencia escolar, así como los motivos de elección de itinerario y especialidad. El tercero interroga sobre las valoraciones y opiniones de las distintas vías posobligatorias y los imaginarios de futuro de los y las jóvenes. Finalmente, el cuarto recoge la composición de la unidad familiar y las ocupaciones y niveles educativos de los adultos y adultas de referencia del alumnado.

Concretamente, en este artículo se analizan las relaciones entre los itinerarios posobligatorios (Bachillerato o CFGM) y las dimensiones de perfil social y expectativas y aspiraciones educativas. Estas dimensiones se han operativizado del siguiente modo:

- Perfil social: Se refiere a la posición que ocupa el alumnado en la estructura social y tiene en cuenta las variables «Clase social», «Género» y «Generación migratoria».

- Clase social: Se conceptualiza la clase social a partir de la categoría profesional y el nivel de estudios más alto presentado por los progenitores

4. Es necesario señalar que esta muestra no es representativa de la distribución del alumnado matriculado en el primer curso de educación secundaria posobligatoria durante el curso 2018-19 en el conjunto de la ciudad de Barcelona. 
del o la alumna. Las categorías profesionales se operativizan a partir del International Standard Classification of Occupation (ISCO-08) y se simplifican en cuatro grupos: clases acomodadas (61), clases medias (553), clases trabajadoras (509) y situaciones de exclusión social (134).

- Género: Se adjudica en función de la respuesta a la pregunta «Indica tu género" y las respuestas válidas se distribuyen entre chicas (762) y chicos (532).

- Generación migratoria: Se construye en función de las trayectorias migratorias protagonizadas por el alumnado y por sus progenitores. Se identifican tres grupos. Los «Autóctonos» (894) son aquellos nacidos y escolarizados en España que descienden de progenitores también nacidos en el país; las «Segundas generaciones» (264) incluyen tanto al alumnado nacido y escolarizado en España descendiente de personas nacidas en otro país como al alumnado nacido fuera de España pero que ha cursado toda la educación obligatoria en el sistema educativo del país; finalmente, el grupo de «Primeras generaciones» (160) incorpora al alumnado nacido fuera de España y no escolarizado en el país antes del inicio de la enseñanza obligatoria.

- Expectativas y aspiraciones educativas: Se identifican los itinerarios formativos esperados y aspirados de los jóvenes a partir de las respuestas a las preguntas «¿Cuál es el nivel educativo más alto que te gustaría alcanzar?» (aspiraciones) y "¿Cuál es el nivel educativo que crees que alcanzarás?» (expectativas).

Para el análisis de las relaciones entre variables, se ha calculado, en primer lugar, la distribución porcentual de las categorías "Clase social», "Género» y «Origen migratorio» en los dos itinerarios, Bachillerato y CFGM. Con ello, se ha podido caracterizar la muestra en términos de perfil social, al tiempo que se han detectado los rasgos principales de su distribución en los dos itinerarios formativos. En segundo lugar, se ha analizado con más detalle la distribución de las expectativas y de las aspiraciones educativas, y la distancia entre unas y otras en función del itinerario educativo posobligatorio y de las variables de perfil social.

\section{Resultados}

\subsection{La composición social de los itinerarios formativos}

El alumnado de los itinerarios académico y profesional está claramente diferenciado tanto en términos de clase social como de generación migratoria (tabla 1). Las diferencias de género, en cambio, tan solo se aprecian en la distribución dentro de cada itinerario, en las especialidades formativas específicas.

El nivel de estudios y la categoría ocupacional de los adultos de referencia difiere significativamente entre el alumnado de Bachillerato y el de CFGM. 
Tabla 1. Distribución del alumnado según itinerario formativo y perfil social

\begin{tabular}{llcc}
\hline & Perfil social & Bachillerato & CFGM \\
\hline Clase social $^{* *}$ & Clases acomodadas & $8 \%$ & $1,2 \%$ \\
& Clases medias & $56,6 \%$ & $29,5 \%$ \\
& Clases trabajadoras & $28,5 \%$ & $54,3 \%$ \\
& Situaciones de exclusión social & $6,9 \%$ & $15 \%$ \\
\hline Generación migratoria $^{* *}$ & Autóctonos & $76,2 \%$ & $58,5 \%$ \\
& Segundas generaciones & $17,2 \%$ & $23,2 \%$ \\
& Primeras generaciones & $6,6 \%$ & $18,4 \%$ \\
\hline
\end{tabular}

* $p \leq 0,05 ;{ }^{* *} p \leq 0,01$. Valor de $V$ de Cramer para la variable «Clase social» $=0,35$. Valor de $V$ de Cramer para la variable «Generación migratoria» $=0,21$.

Fuente: elaboración propia.

En Bachillerato, más de la mitad del alumnado tiene al menos un adulto de referencia con estudios universitarios $(57,7 \%)$. En cambio, entre el alumnado de CFGM este porcentaje es mucho menor (19,3\%). Asimismo, el porcentaje de adultos de referencia con estudios máximos de secundaria obligatoria $o$ posobligatoria es notablemente mayor entre el alumnado de CFGM (56,4 \%) que entre el de Bachillerato (32,3\%).

La categoría ocupacional más presente entre los familiares del alumnado de Bachillerato es «Profesionales científicos e intelectuales» (34,7 \%), que tiene mucho menos peso entre los familiares del de CFGM (10,3 \%). Por otro lado, la categoría con mayor porcentaje entre los adultos de referencia del alumnado de CFGM es la de "Trabajadores de los servicios y vendedores de comercios y mercados» (21,9\%), que dobla el porcentaje obtenido para Bachillerato (11,5\%). Finalmente, el grupo de «Ocupaciones elementales» es minoritario entre las familias del alumnado de Bachillerato (5,5\%), pero mucho más presente en CFGM (12,7\%).

A partir de la agregación de las categorías ocupacionales, se construye la categoría de clases sociales que se usa a lo largo del análisis. Tal y como puede apreciarse en la tabla 1, las clases medias representan más de la mitad de la muestra en Bachillerato, y su proporción es especialmente alta en la especialidad Artística (65\%). En cambio, en los CFGM, la presencia de clases acomodadas es prácticamente residual, y los datos destacan una mayoría de clases trabajadoras y un porcentaje de situaciones de exclusión social significativamente superior al de Bachillerato.

Las diferencias en clave de generación migratoria son claras entre las dos vías formativas. Como se observa en la tabla 1, más de tres cuartas partes del alumnado de Bachillerato es autóctono, mientras que en los CFGM el alumnado autóctono no alcanza el 60 \%. Si nos fijamos en la distribución del alumnado de segundas generaciones, observamos que representa casi una cuarta parte del alumnado de CFGM, mientras que no alcanza el $20 \%$ en Bachillerato. El alumnado de primeras generaciones se distribuye de forma especialmente desequilibrada entre Bachillerato y CFGM (28,7 \% y 71,3\%, respectivamente). 
En clave de género, ${ }^{5}$ en Bachillerato, Ciencia y Tecnología es la única especialidad ligeramente masculinizada, con tan solo un $45 \%$ de alumnas. Las demás especialidades presentan porcentajes de alumnas nunca inferiores al 60 $\%$. En los CFGM, en cambio, la distribución por géneros es más heterogénea. Por un lado, encontramos especialidades altamente feminizadas, como Imagen Personal (86 \% de alumnas chicas), Servicios Socioculturales y a la Comunidad (78 \%) y Sanidad (69 \%). Por el otro, especialidades muy masculinizadas, como Transporte y Mantenimiento de Vehículos (3\%), Informática y Comunicaciones (5 \%) y Actividades Físicas y Deportivas (29 \%). Las únicas especialidades con distribuciones de género equilibradas son Administración y Gestión (55 \%) y Comercio y Marketing (57\%).

Los datos indican, pues, una vía posobligatoria académica formada mayoritariamente por clases medias autóctonas y feminizada en todas las especialidades excepto en la científico-tecnológica. Paralelamente, el perfil social de la vía profesional concentra mayores porcentajes de clases trabajadoras y situaciones de exclusión, y una mayor población de origen migrante que el Bachillerato.

\subsection{El análisis de las expectativas y las aspiraciones}

Como se ha visto, las aspiraciones y las expectativas de los y las jóvenes se distribuyen de forma desigual en función de factores socioeconómicos y estructurales. Por ello, en el análisis que se presenta a continuación exploramos el impacto del itinerario formativo, de la clase social, de la generación migratoria y del género en la distribución de expectativas y aspiraciones entre el alumnado de la muestra. No se trata solo de explorar el efecto aislado de cada una de estas variables, sino también, y sobre todo, de indagar cómo interactúan para contribuir a la definición de distintos horizontes educativos. Nuestro objetivo, entonces, es conocer de qué forma estas variables influyen en la construcción de unos escenarios de futuro que, a su vez, contribuyen en la definición de trayectorias educativas desiguales.

En primer lugar, y atendiendo a la distribución de aspiraciones y expectativas según el itinerario formativo (tabla 2), el elemento que destaca es la clara hegemonía de los estudios universitarios en el horizonte educativo del alumnado de Bachillerato. Así, un 77,7 \% aspira a alcanzar esta titulación una vez finalice la actual etapa. Dicho de otro modo, más de tres cuartas partes de los y las jóvenes que optan por el itinerario académico en la posobligatoria aspiran a cursar posteriormente estudios universitarios. En este sentido, los datos obtenidos apuntan a la consolidación de la idea del Bachillerato como una etapa propedéutica cuyo objetivo primordial es preparar la selectividad. ${ }^{6}$

5. A pesar de que la distribución por especialidades formativas no es el objeto de este artículo, vale la pena destacar que los cálculos realizados para la variable «Género» son significativos aunque la intensidad de la relación es baja ( $\mathrm{p} \leq 0,01$. V de Cramer $=0,084)$.

6. Desde el punto de vista de las funciones que se atribuyen a cada itinerario formativo, este resultado coincide con lo desarrollado por Tarabini y Jacovkis (2019b). 
Por otro lado, en lo relativo al alumnado que actualmente cursa estudios de CFGM, la aspiración de acceder a la universidad cae hasta un 38,5 \%. Esto supone una reducción de más de 39 puntos con relación al alumnado de Bachillerato. De hecho, la aspiración de alcanzar una titulación de Ciclo Formativo de Grado Superior (CFGS) es la más frecuente entre los y las alumnas de CFGM (39,7 \%), aunque muy poco por encima de la aspiración universitaria. Así pues, dentro del itinerario de CFGM se observa un escenario donde conviven dos horizontes de acción: la universidad y los CFGS.

Sin embargo, cuando ponemos el foco en las expectativas (es decir, las previsiones o probabilidades asumidas para un futuro próximo), observamos que la aspiración de obtener una titulación universitaria se reduce en ambos casos, aunque de forma mucho más pronunciada entre el alumnado de CFGM. En el caso del alumnado de Bachillerato, el porcentaje continúa estando cerca del $60 \%$ y el incremento se da sustancialmente en los casos de «No lo sé». Desde nuestro punto de vista, este hecho no manifiesta una reducción real de las expectativas con relación a las aspiraciones, sino que exhibe la mediación que la incertidumbre ejerce en la respuesta dada para las primeras. En este sentido, no es casual que el resto de categorías formativas tenga una variación residual (de poco más de 2 puntos), puesto que la reducción de expectativas no es, en este caso, una reducción como tal.

En el caso del alumnado de CFGM, la situación observada es muy diferente. En este sentido, tan solo un 18,7 \% espera ahora alcanzar una titulación universitaria, y esta es la segunda categoría menos seleccionada de las disponibles, tan solo 1,6 puntos por encima del abandono de la trayectoria formativa una vez finalizados los estudios posobligatorios actuales. De hecho, esta opción emerge con fuerza y crece de un 10,2 \% en las aspiraciones hasta un no menospreciable $17,1 \%$ en las expectativas. La opción de alcanzar una titulación de CFGS aglutina casi un $43 \%$ de las respuestas obtenidas, y es la categoría predominante dentro de las expectativas del alumnado de CFGM. Esto apuntaría al impacto de la estructura del sistema formativo en el horizonte de los y las jóvenes, al consolidar la idea de una doble red formativa: la académica, representada por Bachillerato y universidad, y la profesional, con los CFGM y los CFGS como protagonistas.

En segundo lugar, al introducir el origen social del alumnado encontramos ciertos elementos que es interesante remarcar. Primero, tal y como comentábamos anteriormente, se observa que el alumnado de Bachillerato, independientemente del perfil social, tiene unas aspiraciones y unas expectativas superiores al de CFGM (tabla 3). Por ejemplo, con relación a las aspiraciones, la diferencia entre el alumnado de Bachillerato y el de CFGM a la hora de expresar su voluntad de acceder a la universidad es de 10 puntos entre las clases acomodadas, de 34 puntos entre las clases medias, de 40 puntos entre las clases trabajadoras y de 30 puntos entre el alumnado proveniente de entornos sociales más desfavorecidos. Lo mismo sucede con las expectativas de formalizar dicho acceso. En este caso, las diferencias son de 62 puntos entre las clases acomodadas, de 35 puntos entre las clases medias, de 34 puntos entre las 
Tabla 2. Expectativas y aspiraciones según itinerario formativo

\begin{tabular}{|c|c|c|c|c|c|}
\hline & & $\begin{array}{c}\text { Secundaria } \\
\text { posobligatoria }\end{array}$ & $\begin{array}{l}\text { Título de Ciclo } \\
\text { Formativo de } \\
\text { Grado Superior }\end{array}$ & $\begin{array}{c}\text { Título } \\
\text { universitario }\end{array}$ & No lo sé \\
\hline \multirow[t]{2}{*}{ Bachillerato } & Aspiraciones ${ }^{\star \star}$ & $0,6 \%$ & $6,9 \%$ & $77,7 \%$ & $14,9 \%$ \\
\hline & Expectativas $^{\star \star}$ & $0,3 \%$ & $11,5 \%$ & $57,4 \%$ & $30,8 \%$ \\
\hline \multirow[t]{2}{*}{ CFGM } & Aspiraciones $^{\star \star}$ & $10,2 \%$ & $39,7 \%$ & $38,5 \%$ & $11,5 \%$ \\
\hline & Expectativas $^{\star *}$ & $17,1 \%$ & $42,8 \%$ & $18,7 \%$ & $21,5 \%$ \\
\hline \multirow[t]{2}{*}{ Total } & Aspiraciones ${ }^{\star \star}$ & $5,2 \%$ & $22,4 \%$ & $59,2 \%$ & $13,3 \%$ \\
\hline & Expectativas** & $8,2 \%$ & $26,3 \%$ & $39,1 \%$ & $26,4 \%$ \\
\hline
\end{tabular}

${ }^{*} p \leq 0,05 ;{ }^{* *} p \leq 0,01$. Valor de V de Cramer para la variable «Aspiraciones» $=0,48$. Valor de $V$ de Cramer para la variable «Expectativas» $=0,530$.

Fuente: elaboración propia.

clases trabajadoras y de 20 puntos entre el alumnado en situación de riesgo de exclusión social. Estas diferencias ponen de manifiesto la relevancia del orden escolar y apoyan las hipótesis que afirman que la institución educativa juega un rol importante en la reducción de las expectativas y las aspiraciones de aquellos alumnos que no cumplen los estándares académicos (independientemente de su origen social y, por lo tanto, de las variables contextuales de origen) (Dupriez, et al., 2012; Romito, 2017).

Al mismo tiempo, es posible identificar una tendencia de reducción de las expectativas en función del perfil social, incluso para aquel alumnado que cursa Bachillerato. Por ejemplo, entre los y las jóvenes en la vía académica, la expectativa de acceder a la universidad se sitúa en un 75,9\% para el alumnado de clases acomodadas, y se reduce a un $62,1 \%$ entre el de clase media, a un $50,3 \%$ en el caso del de clase trabajadora y, finalmente, a un 32,6\% entre los jóvenes en situación de riesgo de exclusión social. En este sentido, la diferencia de expectativas entre los que se sitúan en el segmento superior de la estructura social y los que se encuentran en el segmento inferior, a pesar de realizar el mismo itinerario formativo, es de más de 43 puntos. Así pues, pese a que las diferencias son menores que las observadas entre itinerarios, ponen de relieve la importancia que sigue ejerciendo el perfil social a la hora de construir imaginarios y horizontes de acción posibles.

En tercer lugar, en términos de generación migratoria (tabla 4), se observa el mismo patrón de relación entre los itinerarios formativos y los procesos de reducción de las aspiraciones que ha sido señalado anteriormente para la variable "Clase social». Así, con independencia de la generación migratoria, es notable el impacto que tiene el itinerario formativo sobre aspiraciones y expectativas de los jóvenes, así como sobre la distancia entre unas y otras. No obstante, más allá de las tendencias globales, también se identifican algunos elementos específicamente interesantes que apelan a la generación migratoria como variable explicativa. En este sentido, destaca que el alumnado de primeras generaciones, que frecuentemente se encuentra en situaciones sociales más 
Tabla 3. Expectativas y aspiraciones según itinerario formativo y clase social

\begin{tabular}{|c|c|c|c|c|c|c|}
\hline & & & $\begin{array}{c}\text { Secundaria } \\
\text { posobligatoria }\end{array}$ & $\begin{array}{l}\text { Título de Ciclo } \\
\text { Formativo de } \\
\text { Grado Superior }\end{array}$ & $\begin{array}{c}\text { Título } \\
\text { universitario }\end{array}$ & No lo sé \\
\hline \multirow[t]{4}{*}{ Clases acomodadas } & Aspiraciones* & Bachillerato & $0 \%$ & $11,1 \%$ & $81,5 \%$ & $7,4 \%$ \\
\hline & & CFGM & $14,3 \%$ & $14,3 \%$ & $71,4 \%$ & $0 \%$ \\
\hline & Expectativas* & Bachillerato & $0 \%$ & $3,7 \%$ & $75,9 \%$ & $20,4 \%$ \\
\hline & & CFGM & $14,3 \%$ & $42,9 \%$ & $14,3 \%$ & $28,6 \%$ \\
\hline \multirow[t]{4}{*}{ Clases medias } & Aspiraciones ${ }^{\star \star}$ & Bachillerato & $0,3 \%$ & $5,8 \%$ & $79,7 \%$ & $14,2 \%$ \\
\hline & & CFGM & $6,9 \%$ & $32,9 \%$ & $45,7 \%$ & $14,5 \%$ \\
\hline & Expectativas ${ }^{\star \star}$ & Bachillerato & $0 \%$ & $8,4 \%$ & $62,1 \%$ & $29,5 \%$ \\
\hline & & CFGM & $16,2 \%$ & $39,3 \%$ & $27,2 \%$ & $17,3 \%$ \\
\hline \multirow[t]{4}{*}{ Clases trabajadoras } & Aspiraciones ${ }^{\star *}$ & Bachillerato & $1,6 \%$ & $7,3 \%$ & $76,4 \%$ & $14,7 \%$ \\
\hline & & CFGM & $10,4 \%$ & $44 \%$ & $35,5 \%$ & $10,1 \%$ \\
\hline & Expectativas** & Bachillerato & $1 \%$ & $16,8 \%$ & $50,3 \%$ & $31,9 \%$ \\
\hline & & CFGM & $17,6 \%$ & $43,1 \%$ & $16 \%$ & $23,3 \%$ \\
\hline \multirow{4}{*}{$\begin{array}{l}\text { Situaciones } \\
\text { de exclusión social }\end{array}$} & Aspiraciones** & Bachillerato & $0 \%$ & $8,7 \%$ & $67,4 \%$ & $23,9 \%$ \\
\hline & & CFGM & $15,9 \%$ & $36,4 \%$ & $38,6 \%$ & $9,1 \%$ \\
\hline & Expectativas** & Bachillerato & $0 \%$ & $26,1 \%$ & $32,6 \%$ & $41,3 \%$ \\
\hline & & CFGM & $15,9 \%$ & $50 \%$ & $12,5 \%$ & $21,6 \%$ \\
\hline
\end{tabular}

${ }^{*} p \leq 0,05 ;{ }^{* \star} p \leq 0,01$. Valor de V de Cramer para la variable «Aspiraciones» dentro de la categoría "Clases acomodadas" = 0,370; "Clases medias" = 0,433; «Clases trabajadoras" = 0,460; "Situaciones de exclusión social» =0,438. Valor de V de Cramer para la variable «Expectativas» dentro de la categoría «Clases acomodadas" = 0,613; «Clases medias" = 0,537; «Clases trabajadoras" = 0,455; «Situaciones de exclusión social» $=0,404$.

Fuente: elaboración propia.

precarias, es aquel que sufre en menor medida el proceso de reducción de las aspiraciones.

Así pues, y aunque las diferencias son pequeñas y poco significativas (especialmente si se lo compara con el alumnado autóctono), el alumnado de primeras generaciones es el que presenta diferencias porcentuales menores entre aspiraciones y expectativas de acceso y consecución del título universitario (de 20 puntos aproximadamente), con independencia del itinerario formativo que esté cursando. Del mismo modo, esta tipología presenta un menor porcentaje (14\%) de alumnado que realiza actualmente los estudios de CFGM y cree y espera que su trayectoria educativa finalice en la secundaria posobligatoria. Esta cifra se sitúa por debajo tanto del $17 \%$ obtenido para las segundas generaciones como del $18 \%$ obtenido para el alumnado autóctono. Ambos elementos son atribuibles a lo que algunos autores han denominado "optimismo migrante».

7. Este fenómeno describe el hecho de que los estudiantes de origen inmigrante aspiran a carreras educativas más ambiciosas que las que esperan seguir los hijos de los autóctonos de su mismo origen socioeconómico y con un rendimiento escolar comparable (FernándezReino, 2016). 
Tabla 4. Expectativas y aspiraciones según itinerario formativo y generación migratoria

\begin{tabular}{|c|c|c|c|c|c|c|}
\hline & & & $\begin{array}{c}\text { Secundaria } \\
\text { posobligatoria }\end{array}$ & $\begin{array}{c}\text { Título de Ciclo } \\
\text { Formativo de Grado } \\
\text { Superior }\end{array}$ & $\begin{array}{c}\text { Título } \\
\text { universitario }\end{array}$ & No lo sé \\
\hline \multirow[t]{4}{*}{ Autóctono } & Aspiraciones ${ }^{\star \star}$ & Bachillerato & $0,8 \%$ & $6 \%$ & $79,1 \%$ & $14,1 \%$ \\
\hline & & CFGM & $9,4 \%$ & $39,4 \%$ & $38,3 \%$ & $12,9 \%$ \\
\hline & Expectativas ${ }^{\star \star}$ & Bachillerato & $0,4 \%$ & $10,4 \%$ & $60,3 \%$ & $29 \%$ \\
\hline & & CFGM & $17,9 \%$ & $42,1 \%$ & $17,4 \%$ & $22,6 \%$ \\
\hline \multirow{4}{*}{$\begin{array}{l}\text { Segundas } \\
\text { generaciones }\end{array}$} & Aspiraciones ${ }^{\star \star}$ & Bachillerato & $0 \%$ & $10,2 \%$ & $74,1 \%$ & $15,7 \%$ \\
\hline & & CFGM & $10,1 \%$ & $40,6 \%$ & $40,6 \%$ & $8,7 \%$ \\
\hline & Expectativas ${ }^{\star \star}$ & Bachillerato & $0 \%$ & $13,9 \%$ & $45,4 \%$ & $40,7 \%$ \\
\hline & & CFGM & $17,4 \%$ & $43,5 \%$ & $22,5 \%$ & $16,7 \%$ \\
\hline \multirow{4}{*}{$\begin{array}{l}\text { Primeras } \\
\text { generaciones }\end{array}$} & Aspiraciones ${ }^{\star \star}$ & Bachillerato & $0 \%$ & $8,7 \%$ & $68,6 \%$ & $21,7 \%$ \\
\hline & & CFGM & $13,2 \%$ & $39,5 \%$ & $36,8 \%$ & $10,5 \%$ \\
\hline & Expectativas ${ }^{\star \star}$ & Bachillerato & $0 \%$ & $19,6 \%$ & $52,2 \%$ & $28,3 \%$ \\
\hline & & CFGM & $14 \%$ & $43,9 \%$ & $18,4 \%$ & $23,7 \%$ \\
\hline
\end{tabular}

* $p \leq 0,05 ;{ }^{* *} p \leq 0,01$. Valor de V de Cramer para la variable «Aspiraciones» dentro de la categoría "Autóctono» = 0,494; “Segundas generaciones» = 0,434; «Primeras generaciones» =0,409. Valor de V de Cramer para la variable «Expectativas» dentro de la categoría «Autóctono» = 0,558; «Segundas generaciones» = 0,489; «Primeras generaciones» $=0,397$.

Fuente: elaboración propia.

Por último, en términos de género se observa cierta polarización de los resultados obtenidos (tabla 5). En este sentido, se identifica que las chicas presentan mayores aspiraciones de acceso a la universidad, independientemente del itinerario realizado, así como una menor reducción de estas, especialmente en el caso de las que realizan CFGM. Sin embargo, también son las que, desde el itinerario profesional, presentan mayores indicadores de no continuidad de su trayectoria educativa una vez superada la etapa de secundaria posobligatoria (19\%). Por otro lado, los chicos presentan una mayor presencia de los CFGS en su horizonte, especialmente aquellos que cursan un CFGM (47\% frente al $34 \%$ presentado por las chicas). Para finalizar, no se constatan diferencias en clave de género en cuanto a la aparición de procesos de incertidumbre en la consolidación de las expectativas educativas. Así, el porcentaje de «No lo sé» se sitúa en torno al $30 \%$ para los y las jóvenes que cursan Bachillerato y alrededor del $20 \%$ en el caso de los y las jóvenes que realizan CFGM.

En definitiva, los resultados obtenidos ponen de manifiesto dos elementos. En primer lugar, exhiben el papel activo que las variables estructurales (perfil social, origen migratorio y género) juegan en la construcción de determinados y desiguales horizontes de acción. No obstante, también muestran que, en determinados casos, el perfil social o el origen migratorio pueden ejercer como «elementos protectores» frente a la reducción de las expectativas con relación a las aspiraciones. Ello se observa en la menor reducción de expectativas entre el alumnado de clases medias o, de forma contraintuitiva, entre el alumnado de primera generación. En el primer caso, podemos atri- 
Tabla 5. Expectativas y aspiraciones según itinerario formativo y género

\begin{tabular}{|c|c|c|c|c|c|c|}
\hline & & & $\begin{array}{c}\text { Secundaria } \\
\text { posobligatoria }\end{array}$ & $\begin{array}{l}\text { Título de Ciclo } \\
\text { Formativo de } \\
\text { Grado Superior }\end{array}$ & $\begin{array}{c}\text { Título } \\
\text { universitario }\end{array}$ & No lo sé \\
\hline \multirow[t]{4}{*}{ Hombre } & Aspiraciones ${ }^{\star *}$ & Bachillerato & $0,4 \%$ & $6,6 \%$ & $76,4 \%$ & $16,7 \%$ \\
\hline & & CFGM & $8,7 \%$ & $47,3 \%$ & $31,1 \%$ & $12,9 \%$ \\
\hline & Expectativas ${ }^{\star \star}$ & Bachillerato & $0,8 \%$ & $12 \%$ & $56,2 \%$ & $31 \%$ \\
\hline & & CFGM & $14,4 \%$ & $46,2 \%$ & $18,2 \%$ & $21,2 \%$ \\
\hline \multirow[t]{4}{*}{ Mujer } & Aspiraciones ${ }^{\star \star}$ & Bachillerato & $0,2 \%$ & $6,1 \%$ & $79,4 \%$ & $14,2 \%$ \\
\hline & & CFGM & $11,3 \%$ & $33,5 \%$ & $44,8 \%$ & $10,4 \%$ \\
\hline & Expectativas ${ }^{\star *}$ & Bachillerato & $0 \%$ & $10 \%$ & $58,6 \%$ & $31,4 \%$ \\
\hline & & CFGM & $18,8 \%$ & $40,2 \%$ & $19,4 \%$ & $21,7 \%$ \\
\hline
\end{tabular}

* $p \leq 0,05 ;{ }^{* \star} p \leq 0,01$. Valor de $V$ de Cramer para variable «Aspiraciones» dentro de la categoría «Hombre» $=0,537$; «Mujer» $=0,454$. Valor de $\mathrm{V}$ de Cramer para variable «Expectativas» dentro de la categoría «Hombre» = 0,517; «Mujer»= 0,547.

Fuente: elaboración propia.

buirlo al hecho de que las clases medias transitan por un modelo "hecho a medida", objetiva y subjetivamente (Zanten, 2007). En el segundo, a una menor disposición de información sobre la naturaleza selectiva del proceso de acceso a la educación posobligatoria. En segundo lugar, los resultados ponen de manifiesto el papel claro que el orden escolar y la desigual construcción de los itinerarios educativos juegan en este proceso reductor de expectativas. En algunos casos, de hecho, este impacto mediador es incluso más intenso que el de las variables de desigualdad estructural. Así, como se ha mostrado a lo largo del análisis, el impacto del itinerario educativo puede superar al del perfil socioeconómico en la configuración de las expectativas y las aspiraciones de los y las jóvenes.

\section{Conclusión}

Las transiciones a la educación secundaria posobligatoria pueden entenderse como procesos complejos, atravesados por distintos factores estructurales, del sistema educativo y de las propias biografías de los y las jóvenes. Los cambios que están experimentando las pautas de transición y las trayectorias juveniles han sido considerados como procesos de desestandarización a través de los cuales se incrementan los caminos posibles a seguir en la construcción de la trayectoria vital. No obstante, la desestandarización no ha implicado necesariamente un incremento de las oportunidades educativas (Furlong, 2009) y, como hemos visto a lo largo del texto, las desigualdades de carácter estructural y sistémico se mantienen entre el alumnado. Estas desigualdades son visibles tanto en la forma de distribuirse el alumnado entre las vías profesional y académica como en la manera de construir sus expectativas y aspiraciones. En este sentido, los futuros deseados y los futuros previstos por los y las jóvenes 
reflejan una estructura social y un sistema educativo desiguales que les provee de oportunidades educativas y vitales dispares.

A modo de conclusión, en este último apartado señalamos tres cuestiones centrales que emergen de los resultados de nuestro análisis.

La primera remite a la caracterización de la composición de los itinerarios formativos en términos sociodemográficos. Tal y como se muestra en el apartado de contextualización y como ha señalado también la literatura de este campo (Calero, 2008; Tarabini y Jacovkis, 2019b), la composición de los itinerarios académico y profesional refleja desigualdades de género, origen migratorio y perfil social. A pesar del aumento de la oferta en CFGM y de los cambios positivos con relación a su prestigio social, parece claro que las clases medias siguen estando sobrerrepresentadas en el Bachillerato, y las clases trabajadoras en los CFGM. Del mismo modo, la evidencia es abundante al señalar la sobrerrepresentación del alumnado de origen migrado en el itinerario profesional, así como la reproducción de pautas de género en la distribución de chicos y chicas en las distintas especialidades formativas (Lappalainen et al., 2013).

La segunda y la tercera hacen referencia directa al análisis de expectativas y aspiraciones desarrollado en el texto. Por un lado, los datos obtenidos muestran el impacto que tienen las variables sociodemográficas sobre la construcción de escenarios de futuro por parte de los y las jóvenes. Así, hemos visto que el perfil socioeconómico tiene una relación inversa con las expectativas y las aspiraciones, tanto en jóvenes que estudian Bachillerato como en los que estudian CFGM. En este sentido, vemos que las expectativas y las aspiraciones reflejan una estructura social desigual (Curran, 2017). Al mismo tiempo, sostiene la literatura de este ámbito (Dupriez et al., 2012; Ball et al., 2002), expectativas y aspiraciones pueden funcionar como mecanismos para reproducir estas desigualdades al rebajar las metas que los estudiantes se marcan, con independencia de su capacidad para alcanzarlas. En este punto, es preciso señalar la inadecuación de las estrategias de activación y de responsabilización individual a través de las que distintos gobiernos han pretendido aumentar la permanencia de los jóvenes en el sistema educativo y la mejora de su formación para la inserción en el mercado de trabajo (Rodríguez-Soler y Verd, 2018; Serrano y Martín, 2017). Tal y como se ha mostrado en el análisis, es posible detectar pautas de clase, género y origen migratorio en la configuración de las expectativas y de las aspiraciones del alumnado. En este contexto, ¿es adecuado diseñar estrategias de intervención orientadas a aumentar aspiraciones y expectativas sin intervenir en los contextos sociales e institucionales que producen su desigualdad?

Por otro lado, es fundamental señalar el impacto de la arquitectura del sistema educativo y del propio orden escolar sobre los futuros que imaginan los y las jóvenes de los itinerarios académico y profesional. Aunque el análisis presentado no puede discernir con precisión entre los elementos propios de la división por itinerarios (tracking) y los relativos a los procesos que tienen lugar dentro de cada institución, tanto durante la etapa de secundaria obligatoria como durante la etapa posobligatoria, la literatura existente nos da algunas orientaciones que merece la pena señalar. 
En primer lugar, el impacto del itinerario sobre las expectativas y las aspiraciones de los jóvenes por encima del que tiene el resto de variables estructurales analizadas coincide con la evidencia presentada por Lee (2014). El efecto de la separación entre los dos itinerarios formativos no afecta únicamente al alumnado en peor situación socioeconómica, sino que también impacta de forma negativa sobre la equidad del sistema (Hanushek y Wößmann, 2006). Es más, estos trabajos apuntan a un efecto negativo mayor cuanto más temprano se realiza la división de itinerarios. En este sentido, aunque en el caso español esta división se da en un momento relativamente tardío de la trayectoria educativa, es preciso reclamar más atención al desarrollo de una etapa secundaria obligatoria realmente comprensiva, que no premie el conocimiento académico por encima del profesional y marque así las trayectorias educativas en términos de prestigio y utilidad social (Tarabini y Jacovkis, 2019a).

En segundo lugar, es necesario considerar los procesos que se desarrollan dentro de cada institución educativa, tanto en la secundaria obligatoria como en la posobligatoria, pues sus órdenes escolares tienen efectos sobre lo que aspiran y esperan los y las jóvenes. Como sugieren los resultados de este trabajo, y como recoge ampliamente la literatura en el campo de las transiciones y de las trayectorias educativas (Lahelma, 2009; Walther et al., 2015), la institución escolar es un espacio central en la (re)producción de patrones de clase, género y origen migratorio. En este contexto, el papel de docentes y profesionales de la orientación es clave para entender cómo se «enfrían» ${ }^{8}$ las expectativas y las aspiraciones del alumnado. Sea por temor a una frustración posterior por falta de posibilidades materiales para seguir determinados estudios; sea por atribución de mayor o menor capacidad; sea por tratar de evitar una ruptura con los estereotipos de género, o con los deseos familiares; la cuestión es que parece frecuente que las elecciones del alumnado se orienten de acuerdo con un enfriamiento de sus expectativas. Este proceso de enfriamiento no tiene por qué ser voluntario o intencionado para tener un impacto significativo en la interiorización y la reproducción del orden escolar.

A lo largo de este artículo se ha analizado el impacto de variables estructurales y del sistema educativo sobre la construcción de expectativas y aspiraciones por parte de los y las jóvenes de primer curso de la educación secundaria posobligatoria. Sin dejar de lado la importancia de los elementos biográficos que dan sentido a sus trayectorias vitales, es fundamental abordar las desigualdades en los futuros que el alumnado imagina para sí desde el punto de vista de la estructura social y del sistema educativo. Como muestra el análisis presentado, la interacción entre variables del sistema educativo y otras de carácter estructural dibuja escenarios de futuro desiguales para el alumnado que no se agotan con el análisis de cada una de las variables de forma independiente.

8. Tomando las aportaciones de Goffman, la literatura en este campo habla de este proceso como proceso de «cooling out» o enfriamiento de las expectativas (Walther et al., 2015: 357). 


\section{Financiamiento}

Este artículo se ha desarrollado en el marco de la investigación «Edupost16. La construcción de las oportunidades educativas post-16. Un análisis de las transiciones a la educación secundaria post-obligatoria en contextos urbanos» (http://edupost16.es), financiada por el Ministerio de Economía y Competitividad Español para el período 2016-19 (CSO2016-80004-P).

\section{Referencias bibliográficas}

ArCher, Louise; Hollingworth, Sumi; Halsall, Anna (2007). «University's not for $\mathrm{Me}$ - I'm a Nike Person': Urban, Working-Class Young People's Negotiations of Style', Identity and Educational Engagement». Sociology, 41 (2), 219-237. $<$ https://doi.org/10.1177/0038038507074798>.

Armstrong, Patrick Ian; Crombie, Gail (2000). "Compromises in Adolescents' Occupational Aspirations and Expectations from Grades 8 to 10». Journal of Vocational Behavior, 56 (1), 82-98. $<$ https://doi.org/10.1006/jvbe.1999.1709>.

Ball, Stephen J.; Davies, Jackie; David, Miriam; Reay, Diane (2002). "“Classification" and "Judgement": Social class and the "cognitive structures" of choice of Higher Education». British Journal of Sociology of Education, 23 (1), 51-72. $<$ https://doi.org/10.1080/01425690120102854>.

Beal, Sarah J.; Crockett, Lisa J. (2010). «Adolescents' Occupational and Educational Aspirations and Expectations: Links to High School Activities and Adult Educational Attainment». Developmental Psychology, 46 (1), 258-265. $<$ https://doi.org/10.1037/a0017416>.

BecK, Ulrich (1992). Risk Society: Towards a New Modernity. Londres: Sage

Bernardi, Fabrizio; Requena, Miguel (2010). «Desigualdad y puntos de inflexión educativos: el caso de la educación post-obligatoria en España». Revista de Educación, número extraordinario, 93-118.

Biggart, Andrew; Walther, Andreas (2005). «Coping with Yo-Yo Transitions: Young Adults' Struggle for Support, between Family and State in Comparative Perspective». En: LeCCaRdi, Carmen; Ruspini, Elisabetta (eds.). A New Youth?: Young People, Generations and Family Life. Ashgate Publishing.

Blossfeld, Hans-Peter; Shavit, Yossi (2011). «Persisting Barriers: Changes in Educational Opportunities in Thirteen Countries». En: Arum, Richard; Beattie, Irenee R.; ForD, Karly (eds.). The Structure of Schooling. Readings in Sociology of Education. Londres: Sage.

BouRDIEU, Pierre (1973). Cultural reproduction and social reproduction. Londres: Tavistock, 178.

Boxer, Paul; Goldstein, Sara E.; DeLorenzo, Tahlia; Savoy, Sarah; Mercado, Ignacio (2011). «Educational aspiration-expectation discrepancies: Relation to socioeconomic and academic risk-related factors». Journal of Adolescence, 34 (4), 609-617. $<$ https://doi.org/10.1016/j.adolescence.2010.10.002>.

Brunila, Kristiina; Kurki, Tuuli; Lahelma, Elina; Lehtonen, Jukka; Mietola, Reetta; Palmu, Tarja (2011). «Multiple Transitions: Educational Policies and Young People's Post-Compulsory Choices». Scandinavian Journal of Educational Research, 55 (3), 307-324.

$<$ https://doi.org/10.1080/00313831.2011.576880>. 
Calero, Jorge (2008). «Problemas en el acceso a la educación postobligatoria en España». Revista de Sociología de la Educación (RASE), 1 (1), 49-61.

Castejón, Alba; PÀmies, Jordi (2018). «Los agrupamientos escolares: expectativas, prácticas y experiencias». Tendencias Pedagógicas, (32), 49-64. <https://doi.org/10.15366/tp2018.32.004>.

Cuconato, Morena; Walther, Andreas (2015). “"Doing transitions” in education». International Journal of Qualitative Studies in Education, 28 (3), 283-296. <https://doi.org/10.1080/09518398.2014.987851>.

Curran, Marta (2017). «¿Qué lleva a los jóvenes a dejar los estudios? Explorando los procesos de (des)vinculación escolar desde una perspectiva de clase y género». Tesis doctoral. Barcelona: Universitat Autònoma de Barcelona.

Departament D'Ensenyament (2019). «Estadística de l'Ensenyament, curs 2018-19». Barcelona: Departament d'Ensenyament. Último acceso, 15/10/2019. $<$ http://ensenyament.gencat.cat/ca/departament/estadistiques/estadistiques-ensenyament/curs-actual/>.

Dubow, Eric F.; Boxer, Paul; Huesmann, L. Rowell (2009). «Long-term Effects of Parents' Education on Children's Educational and Occupational Success: Mediation by Family Interactions, Child Aggression, and Teenage Aspirations». Merrill-Palmer Quarterly (Wayne State University Press), 55 (3), 224. <https://doi.org/10.1353/mpq.0.0030>.

Dupriez, Vincent; Monseur, Christian; Campenhoudt, Monseur van; Lafontaine, Dominique Marie (2012). «Social Inequalities of Post-secondary Educational Aspirations: Influence of Social Background, School Composition and Institutional Context». European Educational Research Journal, 11 (4), 504-519. <https://doi.org/10.2304/eerj.2012.11.4.504>.

Elias, Marina; Daza, Lidia (2017). «¿Cómo deciden les jóvenes la transición a la educación postobligatoria? Diferencias entre centros públicos y privadosconcertados». Revista de Sociología de la Educación (RASE), 10 (1), 5-22. <https://doi.org/10.7203/RASE.10.1.9135>.

FERnÁndeZ-Reino, Mariña (2016). "Immigrant optimism or anticipated discrimination? Explaining the first educational transition of ethnic minorities in England». Research in Social Stratification and Mobility, 46, 141-156. <https://doi.org/10.1016/j.rssm.2016.08.007>.

Furlong, Andy (2009). "Revisiting transitional metaphors: reproducing social inequalities under the conditions of late modernity». Journal of Education and Work, 22 (5), 343-353. <https://doi.org/10.1080/13639080903453979>.

Furlong, Andy; Biggart, Andy (1999). «Framing "Choices": A Longitudinal Study of Social Aspirations among 13- to 16-year-olds». Journal of Education and Work, 12 (1), 21-36. <https://doi.org/10.1080/1363908990120102>.

Gale, Trevor; Parker, Stephen (2015). «To aspire: A systematic reflection on understanding aspirations in higher education». The Australian Educational Researcher, 42 (2), 139-153. <https://doi.org/10.1007/s13384-014-0165-9>.

Gillborn, David; Rollock, Nicola; Vincent, Carol; Ball, Stephen J. (2012). «You got a pass, so what more do you want?: Race, class and gender intersections in the educational experiences of the Black middle class». Race, Ethnicity and Education, 15 (1), 121-139. $<$ https://doi.org/10.1080/13613324.2012.638869>. 
Hanushex, Eric A.; Wössmann, Ludger (2006). «Does Educational Tracking Affect Performance and Inequality? Differences in Differences Evidence across Countries». The Economic Journal, 116 (510), 63-76. $<$ https://doi.org/10.3386/w11124>.

Hegna, Kristinn (2014). "Changing educational aspirations in the choice of and transition to post-compulsory schooling - a three-wave longitudinal study of Oslo youth». Journal of Youth Studies, 17 (5), 592-613. <https://doi.org/10.1080/13676261.2013.853870>.

Hodkinson, Phil; Sparkes, Andrew C. (1997). "Careership: a sociological theory of career decision making». British Journal of Sociology of Education, 18 (1), 29-44. <https://doi.org/10.1080/0142569970180102>.

KнаттAв, Nabil (2015). «Students' aspirations, expectations and school achievement: What really matters?». British Educational Research Journal, 41 (5), 731-748. <https://doi.org/10.1002/berj.3171>.

Kilpi-Jakonen, Elina (2011). "Continuation to upper secondary education in Finland: Children of immigrants and the majority compared». Acta Sociológica, 54 (1), 77-106. <https://doi.org/10.1177/0001699310392604>.

Lahelma, Elina (2009). «Dichotomized Metaphors and Young People's Educational Routes». European Educational Research Journal, 8 (4), 497-507. <https://doi.org/10.2304/eerj.2009.8.4.497>.

Lappalainen, Sirpa; Mietola, Reetta; Lahelma, Elina (2013). "Gendered divisions on classed routes to vocational education». Gender and Education, 25 (2), 189-205. <https://doi.org/10.1080/09540253.2012.740445>.

LEE, Bommi (2014). «The influence of school tracking systems on educational expectations: a comparative study of Austria and Italy». Comparative Education, 50 (2), 206-228. <https://doi.org/10.1080/03050068.2013.807644>.

Markus, Hazel; Nurius, Paula (1986). «Possible selves». American Psychologist, 41 (9), 954-969.

Rinne, Risto; Silvennoinen, Heikki; Järvinen, Tero; Tiknanen, Jenni (2019). "Governing the Normalisation of Young Adults through Lifelong Learning Policies». En: Parreira, Marcelo Do Amaral; Kovacheva, Siyka; Rambla, Xavier (eds.). Lifelong Learning Policies for Young Adults in Europe. Navigating between Knowledge and Economy. Bristol: Policy Press.

Rodríguez-Soler, Joan; Verd, Joan Miquel (2018). «The design and deployment of the youth guarantee system in Spain. A documentary analysis». Revista Española de Sociología, 27 (3), 395-412. <https://doi.org/10.22325/fes/res.2018.22>.

Romito, Marco (2017). "Governing through guidance: an analysis of educational guidance practices in an Italian lower secondary school». Discourse: Studies in the Cultural Politics of Education, 1-16. <https://doi.org/10.1080/01596306.2017.1314251>.

Serrano, Amparo; Martín, Paz (2017). «From 'Employab-ility' to 'Entrepreneurial-ity' in Spain: youth in the spotlight in times of crisis». Journal of Youth Studies, 20 (7), 798-821. <https://doi.org/10.1080/13676261.2016.1273513>.

Tarabini, Aina; Curran, Marta; Castejón, Alba; Montes, Alejandro (2018). "Framing youth educational choices at the end of compulsory schooling: the 
Catalan case». En: Tarabini, Aina; Ingram, Nicola (eds.). Educational Choices, Transitions and Aspirations in Europe: Systemic, Institutional and Subjective Challenges. Londres: Routledge

TARABINI, Aina; JaCOVKIS, Judith (2019a). «QQué conocimiento para quién? Itinerarios escolares, distribución del conocimiento y justicia escolar». Revista e-Curriculum, 17 (3), 880-908. <https://doi.org/10.23925/1809-3876.2019v17i3p880-908>.

- (2019b). "Transicions a l'educació secundària postobligatòria a Catalunya». En: Riera, Jordi (ed.). Reptes de l'educació a Catalunya. Anuari 2018. Barcelona: Fundació Jaume Bofill.

Thrupp, Martin; Lauder, Hugh; Robinson, Tony (2002). «School composition and peer effects». International Journal of Educational Research, 37 (5), 483-504. $<$ https://doi.org/10.1016/S0883-0355(03)00016-8>.

Tikkanen, Jenni; Bledowski, Piotr; Felczak, Joanna (2015). «Education systems as transition spaces». International Journal of Qualitative Studies in Education, 28 (3), 297-310. $<$ https://doi.org/10.1016/S0883-0355(03)00016-8>.

Walther, Andreas (2006). "Regimes of youth transitions: Choice, flexibility and security in young people's experiences across different European contexts». Young, 14 (2), 119-139. <https://doi.org/10.1177/1103308806062737>.

Walther, Andreas; Warth, Annegret; Ule, Mirjana; Bois-Reymond, Manuela du (2015). "Me, my education and I": constellations of decision-making in young people's educational trajectories». International Journal of Qualitative Studies in Education, 28 (3), 349-371. $<$ https://doi.org/10.1080/09518398.2014.987850>.

Wyn, Johanna; DwYer, Peter (2002). «New Patterns of Youth Transition in Education». International Social Science Journal, 52 (164), 147-159. <https://doi.org/10.1111/1468-2451.00247>.

Zanten, Agnes van (2007). «Reflexividad y elección de la escuela por los padres de la clase media en Francia». Revista de Antropología Social, 16, 245-277. 